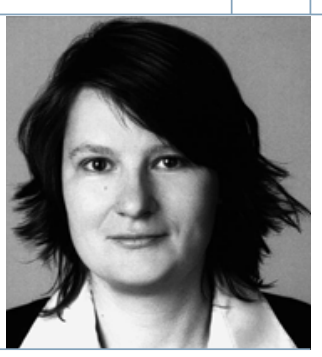

Annegret Wilde

\title{
Wie wichtig ist ein guter Mentor in der Wissenschaft?
}

Mentor ist ein Freund von Odysseus in Homers gleichnamigem Epos. Er sollte dessen Sohn Telemachos zu einem charaktervollen Krieger erziehen. Im Allgemeinen bezeichnet man heute einen erfahrenen Ratgeber als Mentor. Wer auf seinem Weg zu einer wissenschaftlichen Karriere von erfahrenen Kolleginnen und Kollegen begleitet wird, kommt oft einfacher voran. Genauso wichtig wie eine hohe Leistungsbereitschaft ist ein guter Mentor. Man könnte das nicht besser als am Beispiel von Nobelpreisträgern aufzeigen. Schüler von Preisträgern haben nachweislich bessere Chancen, auch sehr erfolgreich zu werden. Viele Nobelpreisträger hatten entweder als Studenten oder jüngere Mitarbeiter mit älteren Nobelpreisträgern zusammen gearbeitet. So erhielten zwei Schüler von Robert Koch, Emil von Behring und Paul Ehrlich, ebenfalls den Nobelpreis.

Aus diesem Grund werden seit etwa 20 Jahren vor allem Mentoring-Programme für Frauen erfolgreich erprobt. Ich selbst bewarb mich 2003 für das Mentoring-Programm der drei Berliner Universitäten, dem ProFiL-Programm (Professionalisierung für Frauen in Forschung und Lehre), das zu den besonders erfolgreichen Programmen gehört, die Frauen auf dem Weg zur Professur fördern. Seit Mitte 2008 ist die Universität Potsdam vierter Kooperationspartner. Von den bisher 317 Teilnehmerinnen des Programms erhielten 89 Frauen bereits einen Ruf auf eine Professur (Stand: Januar 2011). Der Vorteil dieses MentoringProgramms lag für mich vor allem darin, dass ich durch verschiedene Workshops gezwungen war, meine eigene spezielle Situation zu reflektieren und eine realistische Karriereplanung zu entwerfen. Weiterhin war es außerordentlich motivierend, viele Frauen kennenzulernen, die sich auf meinem Qualifizierungsniveau befanden und mit ähnlichen Problemen konfrontiert waren. Über analoge Erfahrungen anderer Teilnehmerinnen berichtete Nature [1]. Der große Erfolg des ProFiLProgramms und auch anderer mittlerweile gut etablierter Mentoring-Programme für Frauen liegt einerseits in der Begleitung der
Mentees durch erfahrene Mentorinnen und Mentoren, andererseits spielt auch der Zugang zu Netzwerken und der Austausch mit anderen Frauen eine große Rolle. Hoch qualifizierte Frauen finden sich in vielen Bereichen (z. B. in den Natur- und Ingenieurwissenschaften) ab einer bestimmten Qualifizierungsstufe meist allein unter Männern - ohne Wissenschaftlerinnen als Vorbild. Warum benötigen Frauen speziell auf sie zugeschnittene Mentoring-Programme, Männer aber nicht? Einigen männlichen Kollegen hätte das ProFiL-Programm ohne Zweifel auch gutgetan. Darum geht es nicht. Mentoren suchen sich vielversprechende Anfänger nach dem Prinzip der habituellen Ähnlichkeiten. Das heißt, unsere meist männlichen Professoren fördern vor allem männliche Kollegen, weil sie ihnen ähnlicher sind. So habe ich Diskussionen erlebt, wer aus dem Kollegenkreis eine begehrte Stelle bekommen sollte, etwa: Kollege X müsse eine Frau und zwei Kinder ernähren, wogegen der Freund von Kollegin Y ja Zahnarzt sei.

Förderung von Personen durch Mentoren ist zu einem erheblichen Teil von der persönlichen Haltung abhängig. Bei der Förderung von Männern durch Männer wird die Motivation meist nicht hinterfragt. Fördert ein Professor aber vor allem Frauen, so muss man meist nicht lange auf Unterstellungen von Kollegen, Mitarbeitern oder Studierenden warten, ob die Förderung möglicherweise außerwissenschaftlich motiviert sei. Auch deshalb sind organisierte Mentoring-Programme, die Mentoren-Beziehungen „demokratisieren“, für Frauen so wirksam. Um promovierende Frauen darin zu bestärken, Karrieremöglichkeiten in der Wissenschaft zu suchen, unterstütze ich die Idee der Frankfurter Zellbiologin Anna Starzinski-Powitz: Das hessenweite Programm SciMento ist mittlerweile eines der größten Mentoring-Programme für Wissenschaftlerinnen in Deutschland. Etwa ein Drittel der SciMento-Mentees sind Biologinnen. Sich als Mentor/in zu engagieren, bringt auch für die eigene Entwicklung sehr viel. So können Mentoren durch ihre Gespräche mit den Mentees ihre eigenen Führungsqualitäten reflektieren, soziale und kommunikative Kompetenzen trainieren und erhalten frische Ideen vom wissenschaftlichen Nachwuchs.

Was zeichnet gute Mentorinnen und Mentoren aus? Sie sollten erfolgreiche Wissenschaftler/innen sein, um den Mentees formelle und informelle Regeln des Wissenschaftsbetriebs näherzubringen. Sie sollten beraten, aber nicht versuchen, den Mentees den eigenen Stempel aufzudrücken. Und ein guter Mentor muss selbst vorleben, was er anderen rät, er muss ein gutes Beispiel sein.

Mentoring als Programm zur Nachwuchsförderung wird in fast allen gesellschaftlichen Bereichen eingesetzt, etwa in großen Unternehmen wie Lufthansa, Deutsche Bank und Telekom. Das hessische Mentorinnen-Netzwerk vermittelt Kooperationen zwischen Studentinnen der naturwissenschaftlichen und technischen Studiengänge und Mentoren aus Wissenschaft und Industrie. Dies fördert die Bildung von karriereorientierten beruflichen Netzwerken, die für Frauen ebenso wie für Männer wertvoll sind.

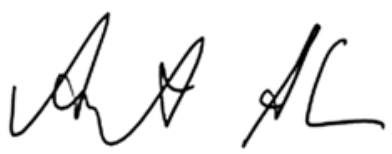

Annegret Wilde

Justus-Liebig-Universität Gießen

\section{Literatur}

[1] Wutte M (2007) Closing the gender gap. Nature 448: 101102

Korrespondenzadresse: Prof. Dr. Annegret Wilde Justus-Liebig-Universität Gießen Institut für Mikro- und Molekularbiologie Heinrich-Buff-Ring 26-32 D-35392 Gießen

Tel.: 0641-9935545

Fax: 0641-9935549

Annegret.Wilde@mikro.bio.uni-giessen.de 